\title{
Sentinel 5 instrument and UV1 spectrometer subsystem optical design and development
}

Jess Köhler, Rik Jansen, Juan Irizar, Alexander Sohmer, Markus Melf, et al.

Jess Köhler, Rik Jansen, Juan Irizar, Alexander Sohmer, Markus Melf, Robert Greinacher, Matthias Erdmann, Volker Kirschner, Abelardo Pérez Albiñana, Didier Martin, Bryan de Goeij, Rob Vink, James Day, Daniël Ten Bloemendal, Wim Gielesen, Jan de Vreugd, Ludger van der Laan, Adriaan van't Hof, "Sentinel 5 instrument and UV1 spectrometer subsystem optical design and development," Proc. SPIE 11852, International Conference on Space Optics - ICSO 2020, 118522M (11 June 2021); doi: 10.1117/12.2599430

SPIE Event: International Conference on Space Optics - ICSO 2021, 2021, Online Only 


\section{International Conference on Space Optics-ICSO 2020}

Virtual Conference

30 March-2 April 2021

Edited by Bruno Cugny, Zoran Sodnik, and Nikos Karafolas
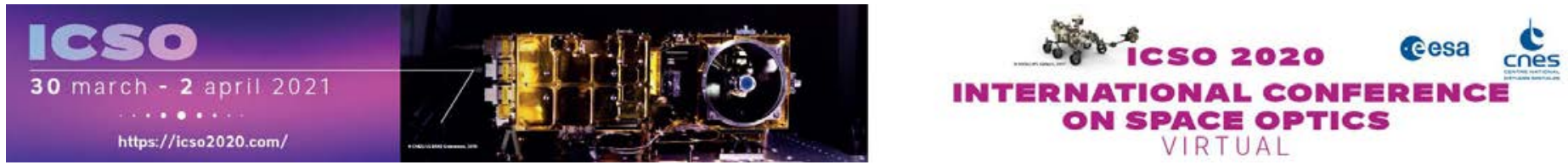

\section{Sentinel 5 instrument and UV1 spectrometer subsystem optical design and development}

\section{Cesa isoporecestings denes}




\title{
SENTINEL 5 INSTRUMENT AND UV1 SPECTROMETER SUBSYSTEM OPTICAL DESIGN AND DEVELOPMENT
}

\author{
Jess Köhler *a, Rik Jansen *c, \\ Juan Irizar a, Alexander Sohmer a, Markus Melf a, Robert Greinacher a, \\ Matthias Erdmann ${ }^{b}$, Volker Kirschner ${ }^{b}$,Abelardo Pérez Albiñana ${ }^{b}$, Didier Martin ${ }^{b}$, \\ Bryan de Goeij ${ }^{c}$, Rob Vink ${ }^{c}$, James Day ${ }^{\mathrm{c}}$, Daniël ten Bloemendal ${ }^{\mathrm{c}}$, Wim Gielesen ${ }^{\mathrm{c}}$, \\ Jan de Vreugd ${ }^{\mathrm{c}}$, Ludger van der Laan ${ }^{\mathrm{c}}$, Adriaan van 't Hof ${ }^{\mathrm{c}}$ \\ a Airbus Defence and Space GmbH, 81663 Munich, Germany; \\ ${ }^{\mathrm{b}}$ European Space Agency, Noordwijk, The Netherlands; \\ ${ }^{c}$ TNO, Stieltjesweg 1, 2628 CK Delft, The Netherlands \\ * jess.koehler@airbus.com, rik.jansen@tno.nl
}

\begin{abstract}
Sentinel-5 is an Earth atmospheric monitoring mission developed within the European Union's Copernicus program. The mission objective is to monitor the composition of the Earth's atmosphere on a daily basis. Airbus DS GmbH acts as the prime contractor under a European Space Agency contract. The instrument design, development, and the instrument verification was and is in many aspects a fruitful co-operation between Airbus and TNO.

The first part of this paper illustrates the optical design of the five optical channels of the Sentinel 5 instrument. The innovative compact optical design sets the basis for the acquisition of the variety of trace gas spectra. The design includes a two free-form mirror wide field telescope, a pointing insensitive Dual Babinet Pseudo Depolariser type polarisation scrambler, three one-dimensional waveguide type homogenizers, five reflective and refractive anamorphotic spectrometers including different disperser types as an a-spherical reflective diffraction grating for the ultra-violet, a grism for mainly the visible, a transmissive grating for the near-infrared, and immersed reflective gratings for the short wave infrared spectral ranges.

The second part of this paper focusses on the design, qualification and verification of the UV1 spectrometer subsystem. Its optical design is based on an Offner-type spectrometer which has been adapted to employ freeform optics and an aspheric off-axis grating. A monolithic housing structure has been developed for optimum stability, accessibility and easy integration of the optical components. The qualification and performance verification of the first Proto Flight Model of the UV1 spectrometer was successfully completed in July 2020.
\end{abstract}

Keywords: Earth Observation, atmospheric monitoring, telescope, spectrometer, homogenizer

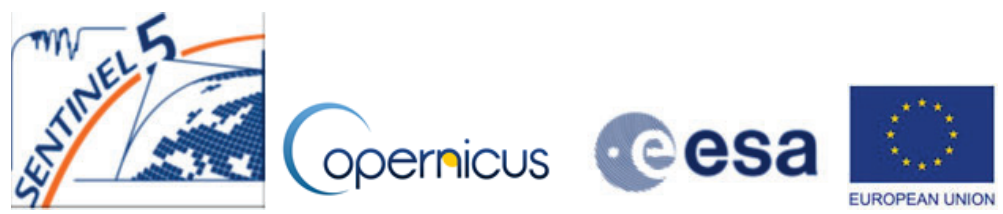




\section{PART I: THE SENTINEL-5 INSTRUMENT}

\section{INTRODUCTION}

The Sentinel-5/UVNS Instrument is an Earth atmospheric monitoring spectrometer developed within the Copernicus program flying on MetOp-SG series of satellites that have been implemented in collaboration between European Space Agency, the European Commission, and EUMETSAT. The mission objective is to monitor the chemical composition of the Earth's atmosphere on a daily basis with the main data products being O3, NO2, SO2, HCHO, CO, CH4 and aerosol optical depth. Airbus Defense and Space $\mathrm{GmbH}$ acts as the prime contractor for the instrument under a European Space Agency contract.

The Sentinel 5 Instrument provides spectroscopic data in several spectral bands from the UV up to the SWIR with different spectral resolution as listed in the table below. See also the publications of Perez Albinana et al [1] and of Irizar et al [2]. Whereas in our previous publication [2] we presented the important and new technological steps of the Sentinel 5 instrument development we focus in this article on the optical design of the complete Sentinel 5 instrument having passed the critical design review. Due to this variety of spectral bands and the different spectral resolutions the spectrometers and the used diffractive dispersers are of different type: A reflective curved grating (UV1), a transmissive grism (UV2-VIS), a transmissive grating (NIR1-NIR2), and immersed gratings (SWIR1, SWIR3).

Table 1. Spectral bands of the Sentinel 5 instrument.

\begin{tabular}{|l|l|l|l|l|}
\hline $\begin{array}{c}\text { Spectral } \\
\text { Band }\end{array}$ & $\begin{array}{c}\text { Minimum } \\
\text { Wavelength }\end{array}$ & $\begin{array}{c}\text { Maximum } \\
\text { Wavelength }\end{array}$ & $\begin{array}{c}\text { Spectral } \\
\text { resolution }\end{array}$ & $\begin{array}{c}\text { Spectrometer of the Sentinel 5 } \\
\text { Instrument }\end{array}$ \\
\hline UV1 & $270 \mathrm{~nm}$ & $325 \mathrm{~nm}$ & $1 \mathrm{~nm}$ & UV1 Spectrometer (UV1SO) \\
\hline UV2 & $300 \mathrm{~nm}$ & $370 \mathrm{~nm}$ & $0.5 \mathrm{~nm}$ & UV2-VIS Spectrometer (U2VSO) \\
\hline VIS & $370 \mathrm{~nm}$ & $500 \mathrm{~nm}$ & $0.5 \mathrm{~nm}$ & UV2-VIS Spectrometer (U2VSO) \\
\hline NIR1 & $685 \mathrm{~nm}$ & $715 \mathrm{~nm}$ & $0.4 \mathrm{~nm}$ & NIR Spectrometer (NIRSO) \\
\hline NIR2 & $745 \mathrm{~nm}$ & $773 \mathrm{~nm}$ & $0.4 \mathrm{~nm}$ & NIR Spectrometer (NIRSO) \\
\hline SWIR1 & $1590 \mathrm{~nm}$ & $1675 \mathrm{~nm}$ & $0.25 \mathrm{~nm}$ & SWIR1 Spectrometer (SWIRSS) \\
\hline SWIR3 & $2305 \mathrm{~nm}$ & $2385 \mathrm{~nm}$ & $0.25 \mathrm{~nm}$ & SWIR3 Spectrometer (SWIRSS) \\
\hline
\end{tabular}

As indicated in Table 1 the UV2-VIS spectrometer combines two spectral bands, the UV2 band and the VIS band, and the NIR spectrometer serves for spectroscopy in the NIR1 and NIR2 band. The SWIR subsystem (SWIRSS) consists of one common collimator optics, a splitter unit followed by two dispersers and two camera optics for the two bands SWIR1 and SWIR3.

The spatial sampling distance for Nadir observation is $7.5 \mathrm{~km}$ for all spectral instrument channels. The spatial oversampling is 6 in the UV1, UV2, VIS, NIR1 and NIR2 spectral channels, which means that six detector pixels cover one spatial sample for these spectral bands. Therefore, spatial information is in principle available down to $1.25 \mathrm{~km}$. However, the spot sizes due to geometrical aberrations of the telescope and the spectrometers are of 40micron at the spectrometer detectors. Due to the pixel size of 20 micron in spatial direction the spatial resolution is of about $2.5 \mathrm{~km}$. For the SWIR1 and SWIR3 spectral bands the spatial oversampling is 3, the spot sizes are in the order of the dimension of one pixel of 15 micron. Therefore, also here the spatial information is available down to approximately $2.5 \mathrm{~km}$.

The polarization insensitivity of the Sentinel 5 instrument is mainly due to the use of a dedicated polarization scrambler present in the telescope intermediate pupil (see the section about the telescope) together with limited polarization sensitivity of the spectrometers. An instrument polarization sensitivity below $0.5 \%$ is achieved on the major part of the field of view.

The instrument contains three different homogenizers: The UV1 homogenizer with a slit width of $0.48 \mathrm{~mm}$, the U2V/NIR homogenizer with a slit width of $0.24 \mathrm{~mm}$ and the SWIR homogenizer with a slit width of $0.248 \mathrm{~mm}$. The homogenizer entrance and exit slit width of the UV1 homogenizer has double size in order to collect more of the low radiance UV light of the Earth spectral scene. The SWIR slit width of $0.248 \mathrm{~mm}$ has been optimized in order to balance the spectral 
oversampling and the spectral resolution for the SWIR1 and SWIR3 spectral channel. All homogenizers have the same length of $9.6 \mathrm{~mm}$. With the $\mathrm{f} \#$ of 10 present in along-track/cross-slit/spectral direction at the homogenizer entrance slit this length results in 0 and $+/-1$ geometrical reflection present in the UV1 homogenizer and $0,+/-1$, and $+/-2$ geometrical reflections in the U2V/NIR and SWIR homogenizers. This already provides sufficient mixing of the specified alongtrack spatial scenes and with this an insensitivity of the instrument spectral response function (ISRF) with respect to a heterogeneous slit illumination. See also the publication of Meister et al [3].

The spatial information for any wavelength of the specified spectral bands needs to be co-registered on all detectors of the instrument to an accuracy of about $10 \%-30 \%$ of the spatial sampling distance depending on the spectral bands. This means that the local viewing directions of two telescopes including homogenizers need to be co-aligned in along-track direction (along track co-registration), that the spectra present at the detectors are straight and co-aligned with detector pixel rows (intra-band co-registration across track), and that the detector pixels for the band centers on different detectors are co-aligned (mean inter-band co-registration across track).

Dedicated means for minimizing the stray-light and a stray-light correction method have been applied in order to fulfill the stringent radiometric accuracy requirements. This relates to the telescope and all spectrometers. We illustrate in more detail the effective straylight counter measures implemented in the UV1 spectrometer subsystem in section 3.

\section{TELESCOPE}

The Sentinel 5 telescope optical design consists of a two mirror wide-field imaging system, a field stop at the intermediate image plane, and a polarization scrambler and aperture stop assembly at the intermediate pupil plane. At the telescope image plane the dichroic beam-splitter and the slit homogenizer are placed providing the input light for the Sentinel 5 five spectrometers.

The field of view angle range of the telescope is of more than $+/-54.2 \mathrm{deg}$ in across-track (act) direction. This is too much for a well-performing polarization scrambler if placed at the telescope entrance pupil. For this reason the polarization scrambler and aperture stop assembly has been placed at the intermediate pupil plane with far smaller angles of incidence. The combination of aperture stop and dual Babinet pseudo-depolariser polarization scrambler with split aperture allows a polarization independent instrument pointing together with a very small polarization sensitivity of the Sentinel 5 instrument.

The field stop at the intermediate image plane limits the field of view of the telescope in across-track direction and provides a stray-light stop for the along-track (alt) direction. The homogenizer entrance slit represents the field stop in along-track direction of the Sentinel 5 instrument. Note that the field of view is curved in the entrance pupil angle space, thus, the along track viewing angle varies with the across track angle. This dependence is also called as telescope smile curve.

The first telescope, the so-called TSBOA VN, provides the light for the UV2-VIS and the NIR1-NIR2 spectrometers. The entrance slit of the U2V/NIR homogenizer is located at the telescope image plane. A dichroic beam-splitter follows the exit slit of the homogenizer and is therefore already part of the UV2-VIS and NIR spectrometer imaging paths.

The second telescope, the so-called TSBOA US, provides the light for the UV1- and the SWIR1-, SWIR3spectrometers. Here, the dichroic is positioned in the telescope optical beam just before the telescope image plane guiding the telescope light towards the UV1 homogenizer and the SWIR homogenizer (part of SWIRSS). The entrance slit width of the UV1 homogenizer has double size compared to the U2V/NIR and the SWIR homogenizer in order to double the path efficiency for the low Earth radiance in the UV1 spectral range. The common UV2-VIS/NIR homogenizer is also in accordance with the smaller specified mis-registration error. 

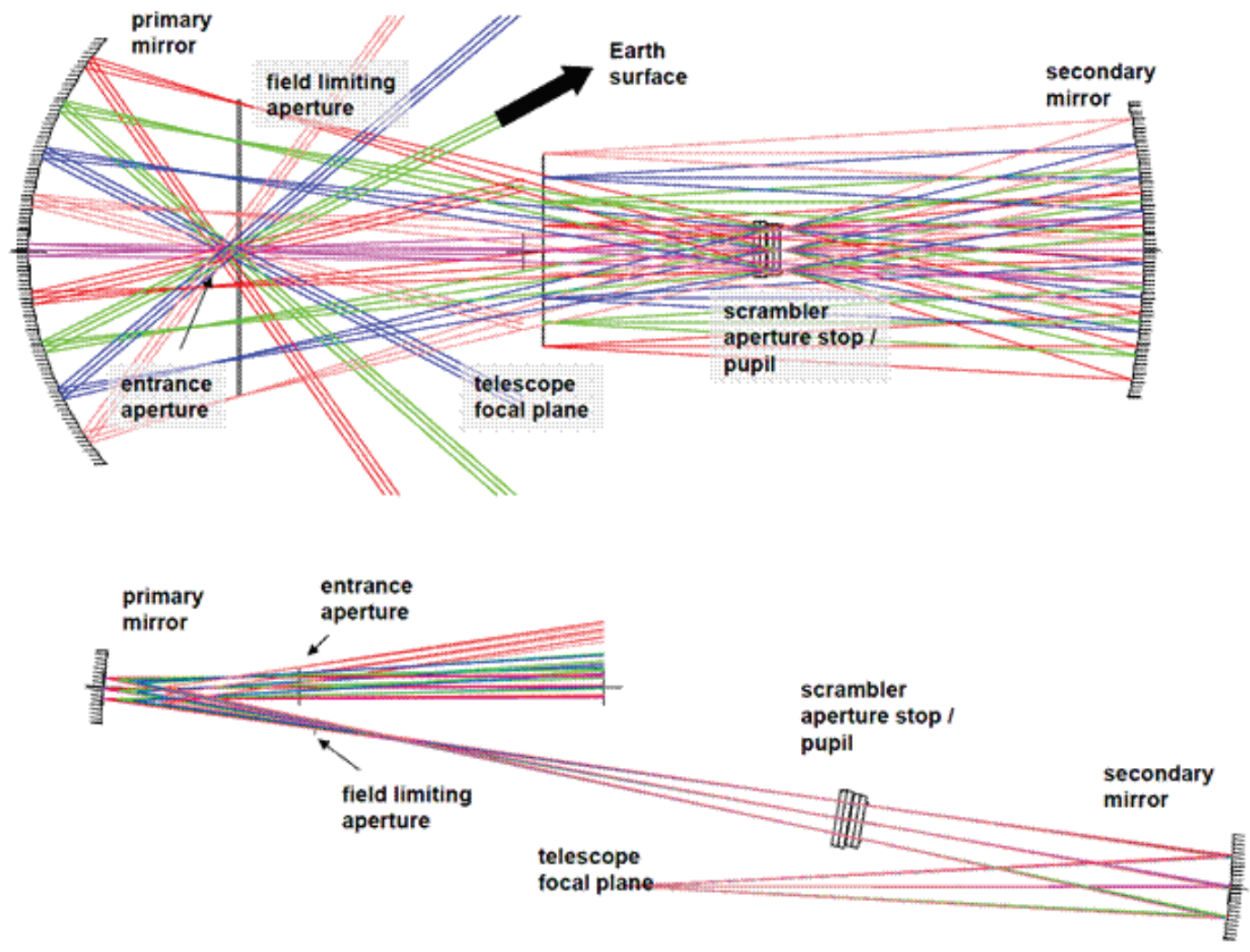

Figure 1. Top and side view of the optical design of the telescope of the Sentinel 5 instrument. The telescope is a wide angle two mirror optical design with accessible entrance pupil, intermediate image and intermediate pupil. A polarization scrambler is placed at the intermediate pupil, an act field stop at the intermediate image, and a diffuser can be positioned at the instrument entrance pupil for spectral and radiometric calibration purposes.
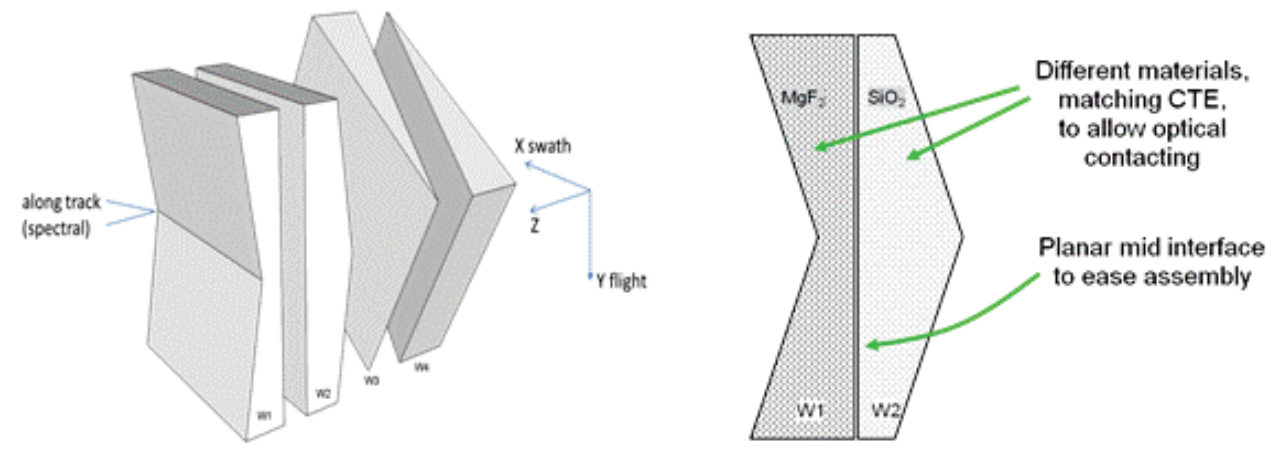

Figure 2. S5 polarization scrambler consisting of two wedge pairs (left; the distance between W1/W2 and W3/W4 is for illustration purpose only). By clever choice of materials with matching CTE, each wedge pair can be optically contacted (right). The V-shaped form of the first wedge pair removes the polarization dependent pointing found in a conventional Dual Babinet Scrambler. The wedge angle is exaggerated for illustration purposes. 
The following figure shows the TSBOA VN hardware. The telescope housing is open on one side close to the instrument entrance pupil (a cover is present in the pictures). This opening is closed by the calibration unit (CAS) that is positioned with respect to the telescope such that the optical elements of the CAS wheel, the aperture stray-light stop, the nominal and reference diffuser, or the deep space mirror can be positioned at the entrance pupil of the Sentinel 5 instrument.

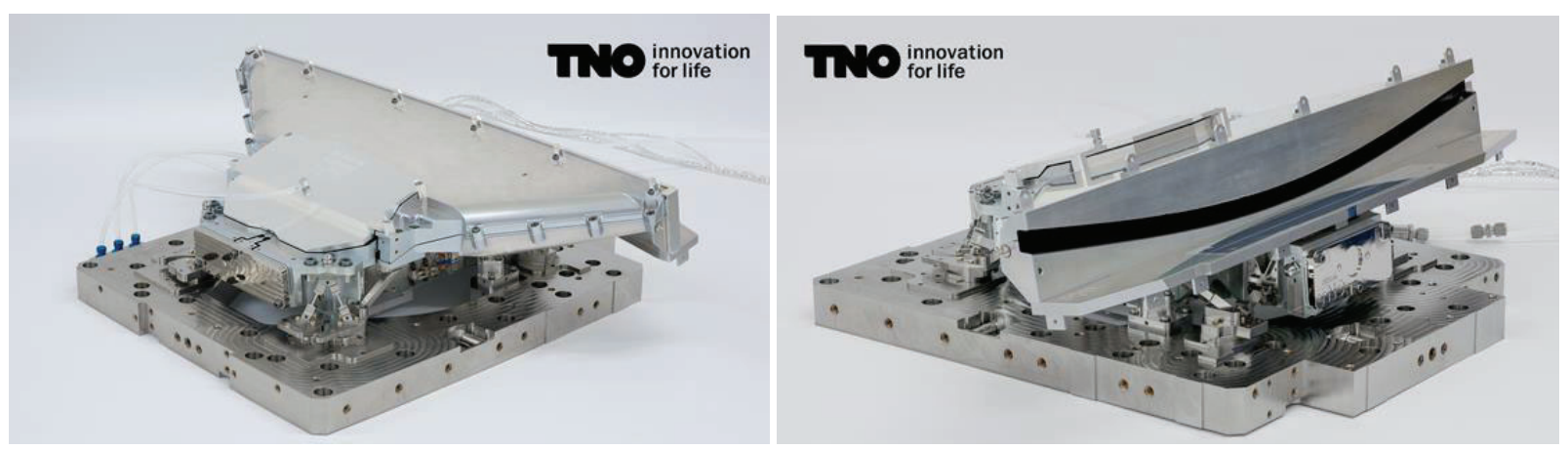

Figure 3. Photos of the Sentinel 5 telescope (TSBOA VN) proto-flight model. The telescopes are provided by TNO in Delft.

\section{UV1 INSTRUMENT CHANNEL}

The UV1 channel of the Sentinel 5 instrument comprises the telescope TSBOA US including the UV1/SWIR beamsplitter used in reflection and the UV1 homogenizer followed by the UV1 spectrometer.

The optical design of the UV1 optical chain of the Sentinel 5 instrument is shown in Figure 4. Concerning the UV1 spectrometer the collimator mirror M1, the curved grating used in reflection, and the camera mirror M2 image the spatial input scene present at the homogenizer entrance slit onto the detector. The collimator mirror has a bi-conical shape in order to compensate the astigmatism introduced by the homogenizer, thus, imaging the spatial information of the homogenizer entrance slit and the uniform distribution in cross-slit direction of the homogenizer exit slit along the spectral direction onto the detector. For a more detailed explanation of the function, the performance, and the reason for the need of the homogenizers within the Sentinel 5 instrument refer to the articles of Irizar et al [2] and Meister at al [3]. The field lens, the frown stray-light correction plate (FSCP), supports the optical distortion correction necessary in order to fulfill the stringent intra-band co-registration requirements.

The two fold mirrors FOLD1, FOLD2 have been introduced in order to place the detector in its focal plane assembly close to the radiator of the instrument. This kind of folding is present in all Sentinel 5 spectrometer optical systems placing the detectors all on one side of the instrument where the radiators are placed. The fold mirror FOLD3 has been introduced for compactness reasons, which is generally a disadvantage of mirror imaging systems.

As disperser a slightly curved conical grating has been introduced providing the necessary dispersion and supporting the optical imaging.

However, the optical imaging is not the driver for this optical design. Instead the strong but rather smooth variation of the spectral input scene radiance of more than 3 orders of magnitude needs dedicated false light counter measures. For this reason the UV1 spectrometer is a mirror design with minimization of ghost images. Also the not used diffraction orders are carefully controlled and guided into absorbing structural elements. In addition, the spectrometer comprises out-of band light suppression mirror coatings on the collimator and first two fold mirrors, so-called "black" coatings as they are black in the VIS spectral range. Furthermore, there is a dedicated spectral filter coating on the rear side of the FSCP that allows only the nominal wavelengths to pass. The optimum position for such a coating is the detector surface itself. Due to the manufacturing difficulty of a considerable layer stack on the CCD sensitive area itself it has been decided to introduce the FSCP with this coating close to the detector. In addition, the mirror surface micro-roughness has been reduced down to below $1 \mathrm{~nm}$ rms in order to minimize the light scattering contribution of the false light. In addition, the grating manufactured by Zeiss Jena with a spectral efficiency between 0.6 and 0.75 is especially optimized for low straylight. 


\section{S5 Instrument UV1 Channel}

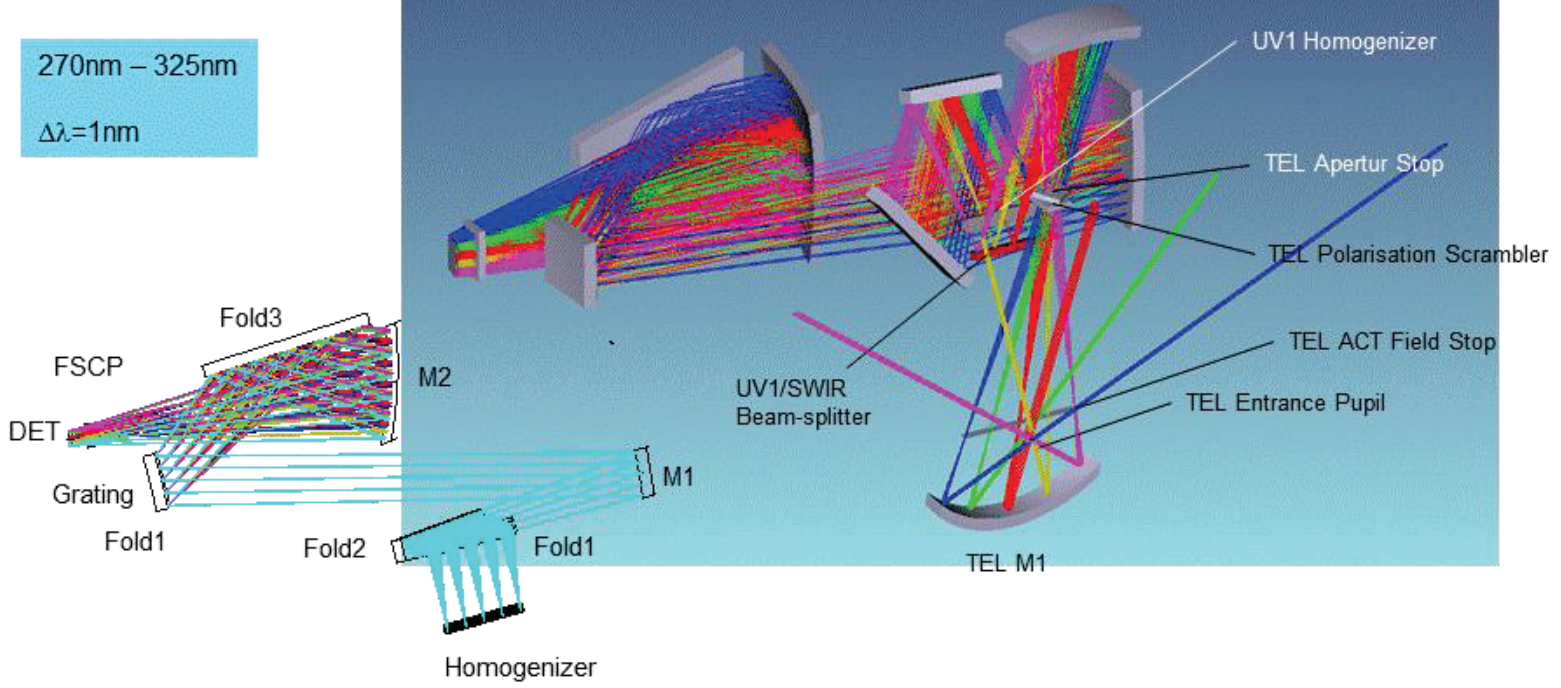

Figure 4. Optical Design of the UV1 spectral channel of the Sentinel5 Instrument. The spectrometer is an Offner-type reflective optical design with a curved grating as disperser.

For the detailed description of the UV1 spectrometer design, development, integration and hardware testing refer to the second part of this article.

\section{UV2-VIS INSTRUMENT CHANNEL}

The UV2-VIS channel of the Sentinel 5 instrument comprises the telescope TSBOA VN including the U2V/NIR homogenizer and the U2V/NIR beam-splitter used in reflection followed by the UV2-VIS spectrometer.

The optical design of the UV2-VIS optical chain of the Sentinel 5 instrument is shown in the figure below. The U2V/NIR homogenizer is common for the UV2, VIS, NIR1 and NIR2 band optimizing the mean inter-band coregistration in along track direction. The UV2-VIS spectrometer optics consists of a refractive astigmatic collimator, a grism as disperser, and a refractive camera lens that is used off-axis.

The first collimator lenses include anamorphotic surfaces necessary for introducing the astigmatism such that the homogenizer entrance surface with the across track spatial information is highly resolved at the detector and the along track spatial information is smeared out.

The two fold mirrors FOLD1, FOLD2 have again been introduced in order to place the detector close to the instrument radiator. Due to the crossed mirror configuration the polarization sensitivity introduced by the two mirrors is mainly compensated.

The dispersing element of the spectrograph generating the spectra is a grism. The grating that is on the rear side of the prism is used in transmission. The incidence and diffraction light directions present at the grating are according to the Littrow condition for a wavelength of $320 \mathrm{~nm}$. Prism and grating parameters have been optimized for minimum slit image distortion (spectrometer smile) at the detector.

Due to the big spectral range and the large Earth radiance differences between the UV2 and the VIS spectral range dedicated means against false light need to be included. Ghost images due to reflections at camera lens surfaces and also at the detector surface have been avoided by the off-axis use of the camera lens and tilt of the detector with respect to the chief-ray orientations. In addition, a linear varying low-pass filter coating has been applied to the flat surface of the last lens which is closest to the detector. This filter coating blocks local out-of band light thus minimizes the cross-talk 
between the high intensity VIS light and the low intensity UV light. Also the detector itself features a similar filter coating. However, due to its simplicity it is less efficient than the one of the last lens surface.

\section{S5 Instrument UV2-VIS \\ Channel \\ $300 \mathrm{~nm}-500 \mathrm{~nm}$ \\ $\Delta \lambda=0.5 \mathrm{~nm}$}

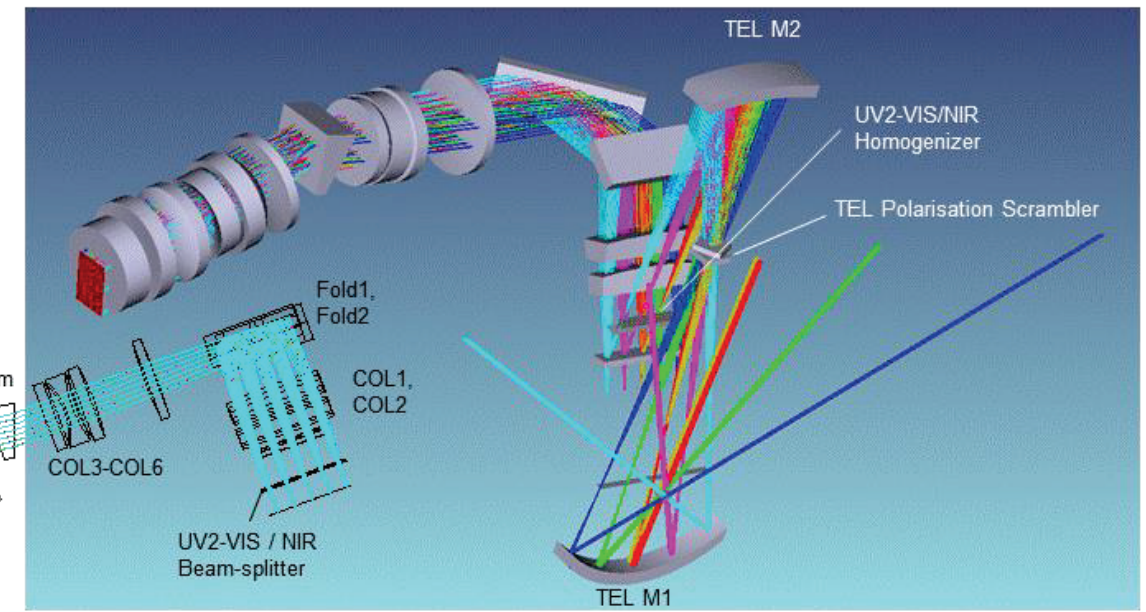

Figure 5. Optical design of the UV2-VIS spectral channel of the Sentinel5 instrument. The spectrometer is a refractive optical design consisting of an astigmatic collimator, a transmissive grism as disperser, a non-symmetrical camera optics used off-axis.

The grating provided by Horiba features a nearly constant efficiency of $0.6-0.75$ within the broad UV2-VIS spectral range and a small polarization sensitivity.

Two pictures of the engineering model of the UV2-VIS spectrometer are shown below. It is representative in terms of mechanical structure, optical mounts, and optical lenses. It will be used for the verification of the stability of the optical image especially under shock loads. The UV2-VIS spectrometer is manufactured and tested by Sodern in Paris.
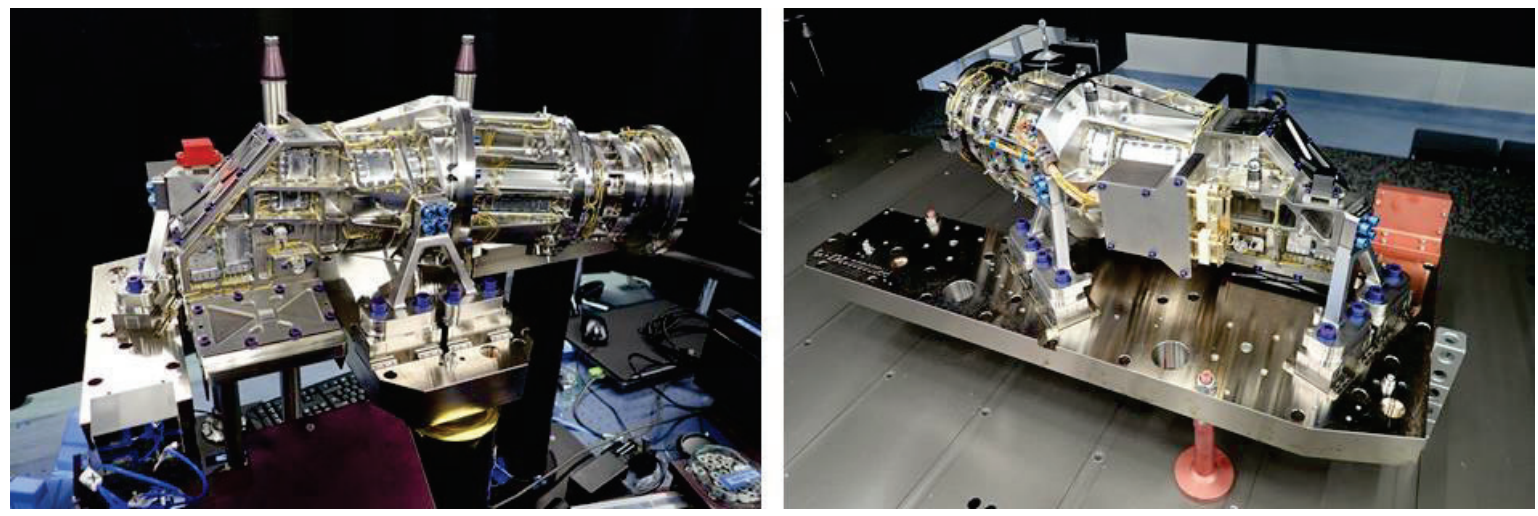

Figure 6. Engineering model of the UV2-VIS spectrometer which is also used for optical/mechanical stability verification before and after shock loads. The UV2-VIS spectrometer is provided by Sodern, Paris.

\section{NIR1-NIR2 INSTRUMENT CHANNEL}

The NIR channel of the Sentinel 5 instrument comprises the telescope TSBOA VN including the U2V/NIR homogenizer and the U2V/NIR beam-splitter used in transmission followed by the NIR spectrometer. 
The optical design of the NIR optical chain of the Sentinel 5 instrument is shown in the figure below. The U2V/NIR homogenizer is common for the UV2, VIS, NIR1 and NIR2 bands optimizing the co-registration in along track direction. The NIR spectrometer optics consists of a refractive anamorphotic collimator, a prism for beam folding and for compensation of aberrations, the refractive grating element, and an on-axis rotational symmetric refractive camera lens. Spectra of the full spectral range between $685 \mathrm{~nm}$ (NIR1 lower boundary) and 773nm (NIR2 upper boundary) are present on the detector.

The first collimator lenses include anamorphotic surfaces necessary for imaging the spatial and spectral object planes separated by the homogenizer length of $9.6 \mathrm{~mm}$ onto the image plane at the detector. This means that the across-track or spatial information present at the telescope image plane is highly resolved whereas the along track spatial information is scrambled since for this direction the homogenizer exit plane is imaged onto the detector.

The two fold mirrors FOLD1, FOLD2 have again been introduced in order to place the detector close to the instrument radiator. Due to the crossed mirror configuration the polarization sensitivity introduced by the two mirrors is mainly compensated.

As disperser a transmissive grating in Littrow configuration is used. Due to the large diffraction angles the optical smile distortion is considerable. This is mainly compensated by the refraction at the surfaces of the prism.

\section{S5 Instrument NIR Channel}

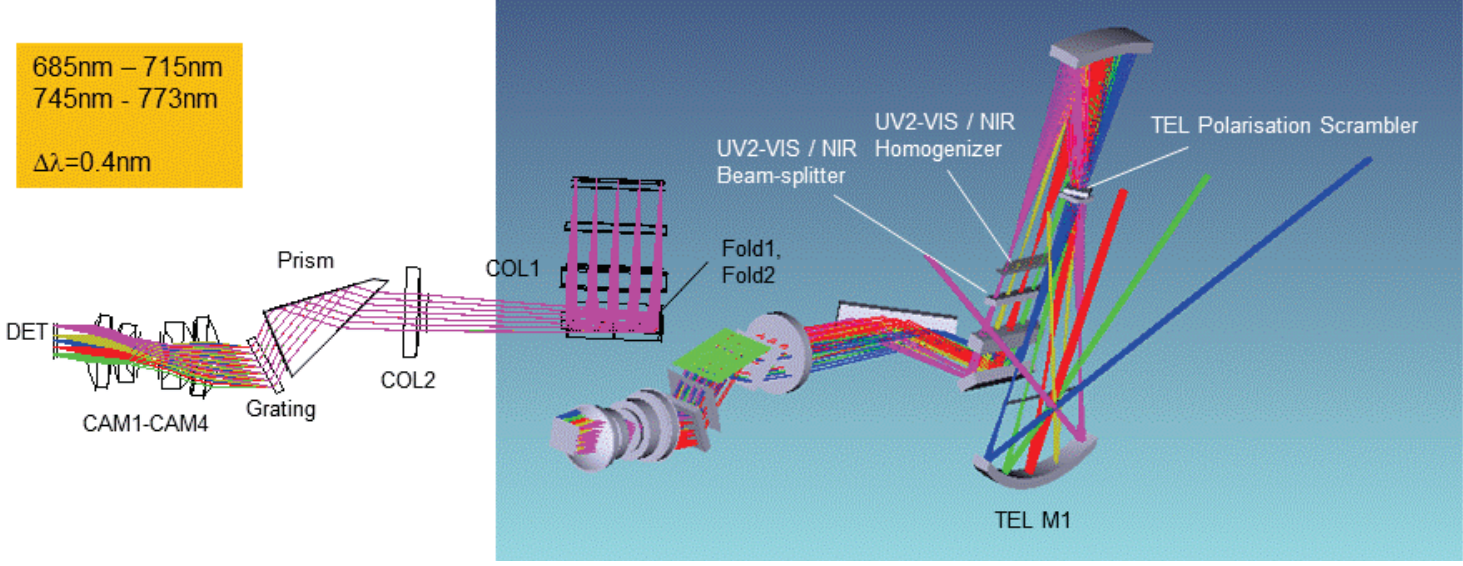

Figure 7. Optical Design of the NIR1-NIR2 channel of the Sentinel5 Instrument. The spectrometer is a refractive optical design with an astigmatic collimator, a transmissive grating as disperser, and an on-axis rotational symmetric camera lens.

The spectral efficiency of the grating has been optimized for the NIR spectral range yielding values between $93 \%$ - $98 \%$ and with this also a small polarization difference. The grating is provided by IOF in Jena.

The figure below shows the NIR spectrometer structural model (SM). Since the spectrometer structure, the optical mounts, and the optical elements are manufactured as for a flight model - the less performing elements have been implemented - and as this model is used also for optical testing and optical stability verification, it could also be an engineering model. The NIR spectrometer is manufactured and tested at Jena Optronics. 


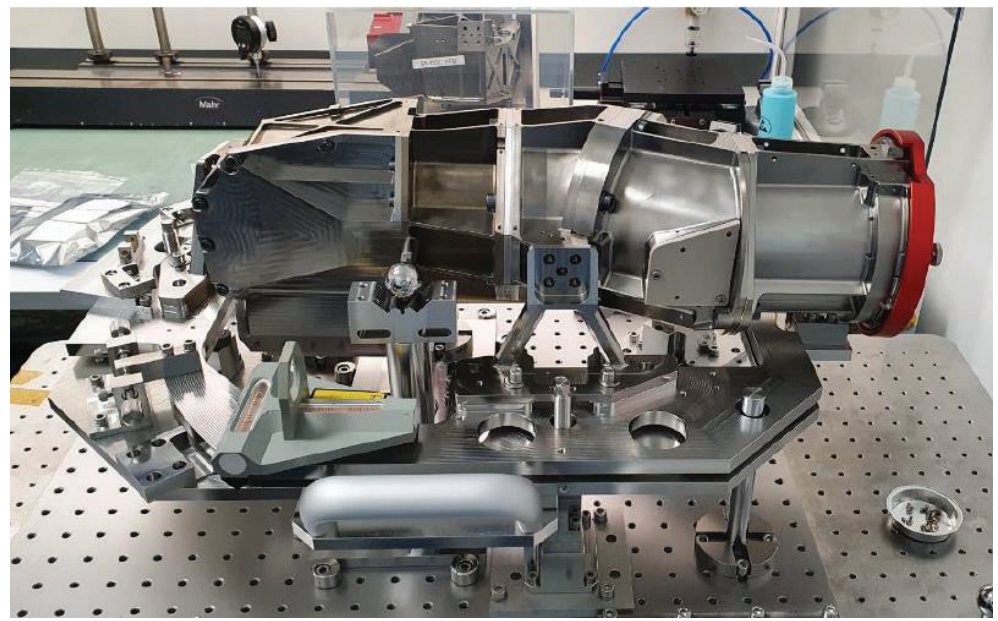

Figure 8. NIR spectrometer SM. Although a structural model it includes flight representative structure, lens mounts, and the less performing optical components. It is used for verifying the stability of the optical imaging also under shock load and for testing the optical verification tools. The NIR spectrometer is provided by JOP Jena.

\section{SWIR1-SWIR3 INSTRUMENT CHANNELS}

The SWIR1 and SWIR3 channels of the Sentinel 5 instrument comprises the telescope TSBOA US including the UV1/SWIR beam-splitter used this time in transmission and the SWIR subsystem that includes the SWIR homogenizer followed by the SWIR collimator optics, the dichroic splitter unit, the two immersed gratings and the separate camera optics of the SWIR1 and SWIR3 spectrometers.

The optical design of the SWIR1 and SWIR3 optical chains of the Sentinel 5 instrument is shown in the figures below. The refractive collimator optics includes a first anamorphotic lens that introduces the astigmatism necessary for compensating the different spatial and spectral object plane locations at the homogenizer entrance and exit surfaces. The collimated light beam passes the dichroic beam-splitter separating the SWIR1 and the SWIR3 spectral bands. A prism is used for beam-folding introducing aberrations that compensate those of the immersed grating following in the optical path. The immersed grating is a prism with a grating at the reflective rear side. The double refraction at the prism front surface together with the grating used in $5^{\text {th }}$ (SWIR1) and $6^{\text {th }}$ diffraction order (SWIR3) allows for the very high dispersion necessary to achieve the required small spectral resolution of $0.25 \mathrm{~nm}$. The plane parallel refractive element just in front of the immersed grating and thus used twice in the optical path represents a spectral band-pass filter limiting the SWIR1 and SWIR3 spectral range. Rotational symmetrical refractive camera lenses focus the dispersed light onto the detectors generating the straight spectra for the various field positions.

Also for the SWIR spectral ranges the Earth spectral scenes with the deep spectral absorption peaks require a minimization of false light. This means small surface roughness of the optical surfaces, minimized particulate contamination, suppression of out-of band light including neighboring diffraction orders, and a dedicated ghost image optimization.

Generally, the refractive optical system has the disadvantage of ghost images due to lens/detector surface double reflections. For the Sentinel 5 instrument this refractive type of optical design was chosen due to its compactness. At the same time this means introducing ghost image counter measures as dedicated anti-reflex coatings on the lens surfaces, and also at the SWIR detector surface itself where the reflection is generally a major contributor to this kind of false light.

In addition, the optics of the SWIRSS is at cold temperatures of about $190 \mathrm{~K}$ in order to minimize self-emission contributions. Cold shields are implemented at and close to the intermediate pupil plane in order to minimize the view to the warmer aperture stop present in the telescope. 


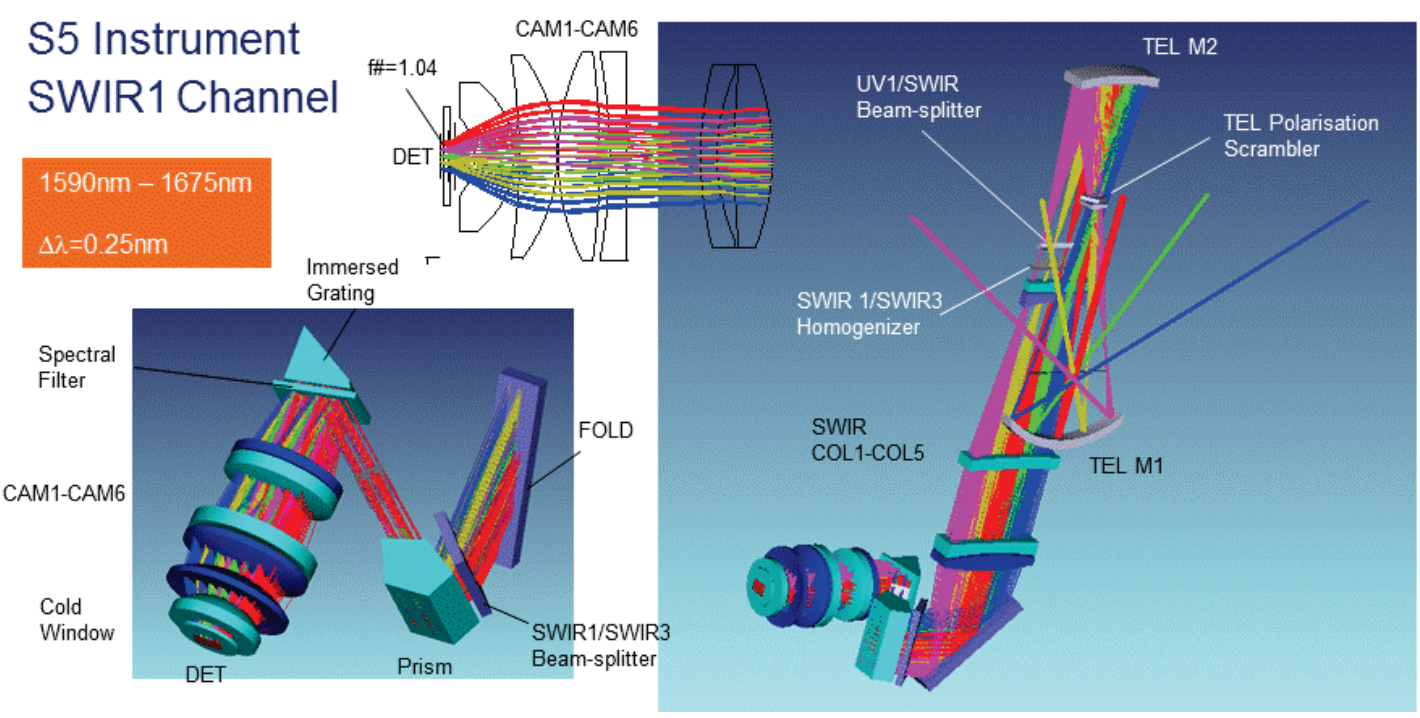

Figure 9. Optical Design of the SWIR1 spectral channel of the Sentinel 5 Instrument. The spectrometer is a refractive optical design with an astigmatic collimator, an immersed grating as disperser, and an on-axis camera lens.

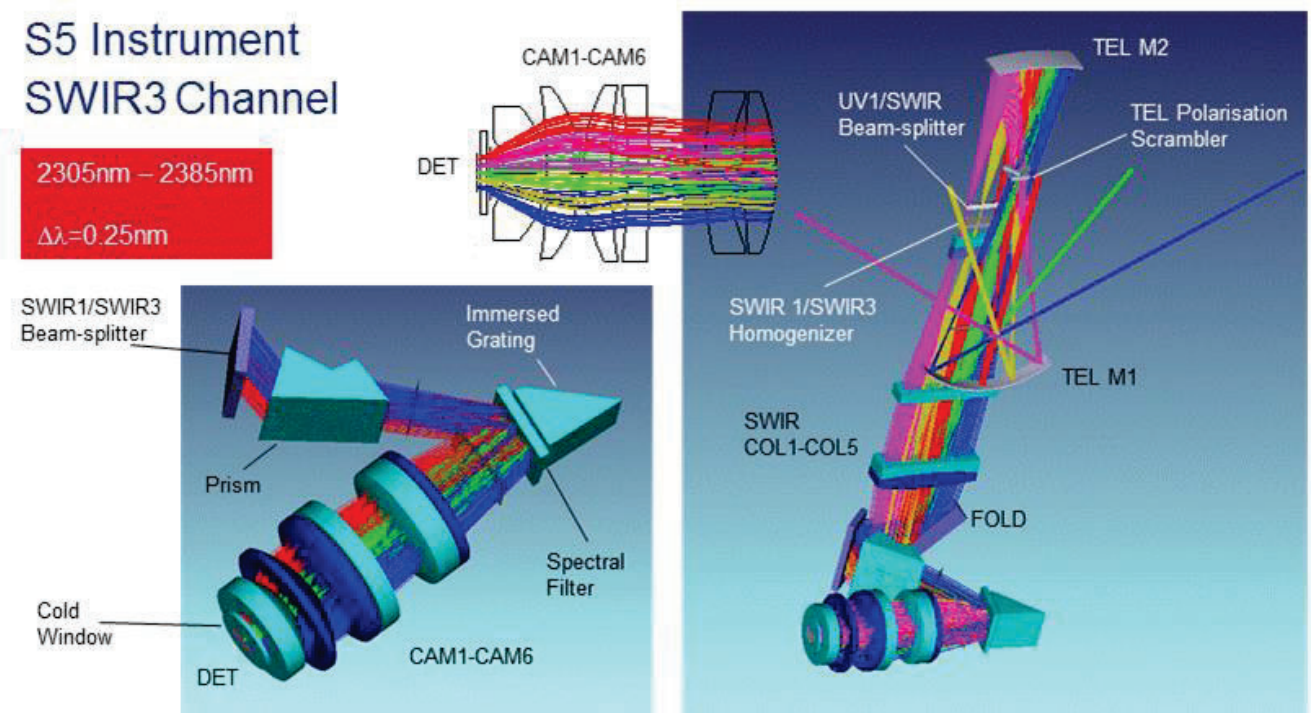

Figure 10. Optical Design of the SWIR3 spectral channel of the Sentinel5 Instrument. The spectrometer is a refractive optical design with an astigmatic collimator, an immersed grating as disperser, and an on-axis camera lens.

\section{CURRENT STATUS}

Currently the Sentinel 5 instrument is in its proto-flight model production phase: Some optical subsystems have just been delivered to the prime, some are going to be delivered soon, some are currently aligned, or are during the testing campaigns. This also means that the instrument integration and alignment phase is currently starting with the mechanical, electrical, and optical ground support equipment being ready or in its finalization phase. In fact the UV1 spectrometer provided by TNO was the first subsystem that had been delivered to Airbus in 2020. Characterizations of the instrument alignment and testing tools, of the instrument structure and of the first in-coming subsystems are currently done. In the course of the optical support program we expect early knowledge of the optical performance of the Sentinel 5 protoflight model. 


\section{PART II: THE UV1 SPECTROMETER}

\section{THE UV1 SPECTROMETER DESIGN}

\subsection{Main system requirements and design challenges}

The main performance requirements of the UV1 Spectrometer subsystem are listed in Table 1. Reaching these performance requirements for a space-born imaging spectrometer is in itself a major challenge. However, the UV1 subsystem has a few more specific development challenges:

1. Tight volume and mass requirements.

The limited volume available increases the complexity of the free-form optical surfaces. Furthermore the distance between the nominal light paths and the housing has to be kept to a minimum so great care needs to be taken to avoid ghosting and stray light from the mechanics. Finally, the housing needs to be thin and lightweight but it also needs to fulfill the stringent mechanical requirements, which leads to a complex mechanical design.

2. The telescope, entrance slit, and detector are part of other subsystems.

This means that the two most important reference systems of the UV1 are virtual points in space, complicating the assembly, integration and verification. Additionally, the illumination, the entrance slit and the detector need to be mimicked by the UV1 Ground Support Equipment (GSE).

3. Tight knowledge requirements on reference systems.

Because the Sentinel-5 instrument is a modular system, the knowledge requirements on the position and orientation of the interface reference systems are typically $<10 \mu \mathrm{m}$ and $<100 \mu \mathrm{rads}$, including Ground-to-orbit effects. Measuring these - sometimes virtual - reference systems with such an accuracy is a major challenge for the Ground Support Equipment (GSE) and the performance verification program.

4. Straylight

Because of the large variation ( $>3$ orders of magnitude) in Earth radiance across the UV1 spectrum, there are strict requirements on in-band and out-of-band straylight. This leads to challenging roughness requirements on the mirrors and the grating, specific coatings that suppress all wavelengths that are not used and also a structure configuration that employs appropriate baffling.

Table 2. UV1 subsystem main performance requirements

\begin{tabular}{|l|l|l|}
\hline Requirements & Value & Remarks \\
\hline Wavelength range & $270-310 \mathrm{~nm}$ & $\begin{array}{l}\text { Performance to be met over this range. Transmission } \\
\text { required up to 325 nm for calibration }\end{array}$ \\
\hline Spectral resolution & $0.97 \mathrm{~nm}$ & $\begin{array}{l}\text { Spectral and spatial slits separated axially by } 9.6 \mathrm{~mm} \\
\text { astigmatic object) }\end{array}$ \\
\hline Slit width (spectral $\times$ spatial) & $0.48 \times 65 \mathrm{~mm}$ & Rectangular aperture, telecentric object \\
\hline Object F/\# (spectral $\times$ spatial) & $10 \times 9$ & $\begin{array}{l}\text { Also requirements on variation of image quality over } \\
\text { field and wavelength }\end{array}$ \\
\hline Image size (spectral $\times$ spatial) & $<8.85 \times<26.65 \mathrm{~mm}$ & Spectral distortion as a function of across-track field \\
\hline $\begin{array}{l}\text { RMS spot diameter }(\text { spectral } \\
\times \text { spatial) }\end{array}$ & $<47 \times<37 \mu \mathrm{m}$ & Spatial distortion as a function of wavelength \\
\hline Smile & $>45 \mu \mathrm{m}$ & Spatial distortion as a function of across-track field \\
\hline Frown & $<6 \mu \mathrm{m}$ & $<1 \%$ \\
\hline Spatial distortion & $<0.3 \%$ (in-band scattering) & $\begin{array}{l}\text { Out-of-band straylight from 200-270 nm and 310 to } \\
1200 \text { nm }\end{array}$ \\
\hline Straylight & $<0.2 \%$ (in-field ghost) \\
& $<0.3 \%$ (out-of-band) & \\
\hline Polarization sensitivity & $<18 \%$ & \\
\hline
\end{tabular}




\subsection{Optical design}

The UV1 spectrometer optical design was realized in close collaboration between Airbus Defence and Space and TNO and is shown in Figure 11. Three wavelengths are shown in this image: $270 \mathrm{~nm}$ (blue), $290 \mathrm{~nm}$ (green) and $310 \mathrm{~nm}$ (red). Three across-track field positions are shown (nadir and \pm full swath).

The Sentinel 5 telescope subsystem (TSBOA) images a curved line on the earth onto a slit-homogenizer, which homogenizes the illumination in the along-track direction. In doing so, the spatial and spectral objects of the UV1 spectrometer are axially separated. This object is collimated, dispersed and re-imaged onto a 2D detector, such that the spatial and spectral information can be retrieved. The spectrometer is based on an Offner-type spectrometer where all powered components share one optical axis. The classical Offner design with spherical components and unity magnification is adapted to employ freeform optics and to generate a magnification of 0.4 . This configuration in principle leads to a design with low aberrations and low distortion.

The spectrometer starts at two axially-split slit planes for the across-track and along-track dimensions. Two fold mirrors (Fold 1 and Fold 2) direct the light into the spectrometer plane of symmetry. Fold 1 and Fold 2 are rotated about orthogonal axes for polarization control. A single collimator mirror ( $\mathrm{Col} \mathrm{M} 1$ ) collimates the light and directs the light towards the grating. The grating disperses the spectrum and generates a virtual slit image. This image is re-imaged by the mirror Cam M2 onto the detector. Fold 3 is a flat fold mirror that is used to minimize the volume of the unit. In front of the detector, a frown-stray-light-correction plate (FSCP) is used. This field lens reduces the frown (keystone distortion) and has a graded coating on the planar surface to improve stray light performance.

The anamorphicity of the UV1 aperture, the astigmatism of the UV1 object planes and the restrictive packaging constraints lead to a design in which the powered mirrors are off-axis aspherics. To realize a thermo-mechanically stable construction, both housing and mirror bodies are made from aluminium thereby realizing an isothermal design. The three folding mirrors and two free-form mirrors are manufactured by AMOS, Belgium. The mirrors have stringent specification on form and slope error and the micro-roughness specification is $<0.7 \mathrm{~nm} \mathrm{rms}$.

The grating is a convex conic that is used off-axis. Its line spacing is constant in projection and is blazed to stringent straylight, transmission and polarization sensitivity requirements in the UV1 region. The grating substrate is fused silica with an aluminium top layer and is made using a holographic lithographic process by Carl Zeiss GmbH, Germany [4]

Finally, the Frown Stray Light Correction plate (FSCP) is a fused silica field lens with a planar back surface to support a graded coating for straylight suppression. Its front surface is defined to correct frown, i.e. it should ensure that the spectrum from one spatial point in the entrance slit is imaged as a straight line on the detector. The FSCP substrate is manufactured by Optimax, USA.

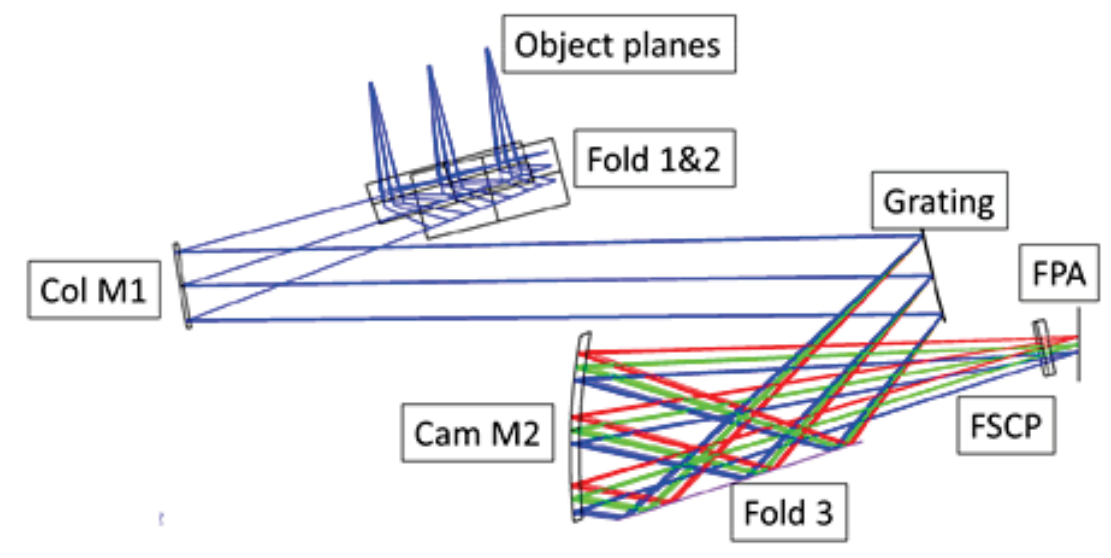

Figure 11. Optical design of the UV1 SO showing the chief and marginal rays at 3 across-track field positions (nadir and \pm full swath) and 3 wavelengths $270 \mathrm{~nm}$ (blue), $290 \mathrm{~nm}$ (green) and $310 \mathrm{~nm}$ (red)

\section{Coatings}

The mirror and lens coatings for the UV1 spectrometer fulfill multiple functions. Firstly, the coatings in combination deliver an in-band system transmission from $270 \mathrm{~nm}$ to $325 \mathrm{~nm}$ that varies from $\sim 50 \%$ at short wavelengths to $\sim 2 \%$ at 
long wavelengths. Secondly, these mirror coatings in combination have a polarization sensitivity that is approximately complementary to the polarization sensitivity of the grating, so that the entire system is compliant to the polarization sensitivity requirement. Finally, the coatings are designed to absorb out-of-band Earth light, with an optical density of $>5$ from 340 to $1200 \mathrm{~nm}$. This optical density at out-of-band wavelengths is achieved by applying an absorbing coating beneath the dielectric stacks on four of the five mirrors.

In addition to the AR coating on the first surface of the FSCP, the second surface of the FSCP supports a graded coating. This coating is a dielectric stack that acts as a short-pass filter. The coating thickness is tapered in the spectral direction across the FSCP. This taper causes the cut-on wavelength to shift across the surface of the lens at the same rate as the dispersed spectrum. This filter therefore always transmits nominal light but reflects wavelengths that are approximately $15 \mathrm{~nm}$ longer than nominal. The justification for this coating and the absorbing coatings on the mirrors is given in the straylight section below. All coatings (except for the grating) are designed and manufactured by Optics Balzers Jena $\mathrm{GmbH}$, Germany under management from AMOS [5].

Stray light

Both the in-band and out-of-band straylight requirements for the UV1 spectrometer are challenging, mainly because the Earth radiance varies by more than three orders of magnitude over the UV1 wavelength range. The in-band straylight performance is dominated by the scattering from microroughness on the optics, with a small contribution from scattering from particle contamination. A market consultation at the start of the project revealed that the obtainable roughness values with today's state-of-the art manufacturing technologies were about $0.7 \mathrm{~nm}$ for the aluminium mirrors and about 2 $\mathrm{nm}$ for the grating. In combination with all the other stray light prevention measures these roughness values limit the achievable stray light fractions to approximately $2 \%$.

\subsection{Mechanical design}

The mechanical design of the UV1 spectrometer is mainly driven by requirements on strength, stability and dynamic performance. The design process is mainly constrained by the optical lay-out, design-envelope, minimal eigenfrequency of $180 \mathrm{~Hz}$ and a maximum mass of $9.5 \mathrm{~kg}$. An overview of the mechanical design is given in Figure 12. The UV1 mirrors are provided with flexures to minimize the impact of integration loads on the Surface Form of the optical surface. The grating and FSCP are adhesive bonded to mounts with integrated leaf springs.

The UV1-housing is an aluminium thin walled structure with a thickness of $1 \mathrm{~mm}$. At the interfaces where optical components are positioned, stringent mechanical tolerances of $5 \mu \mathrm{m}$ are applied to prevent distortion of the surfaces of the optical components.
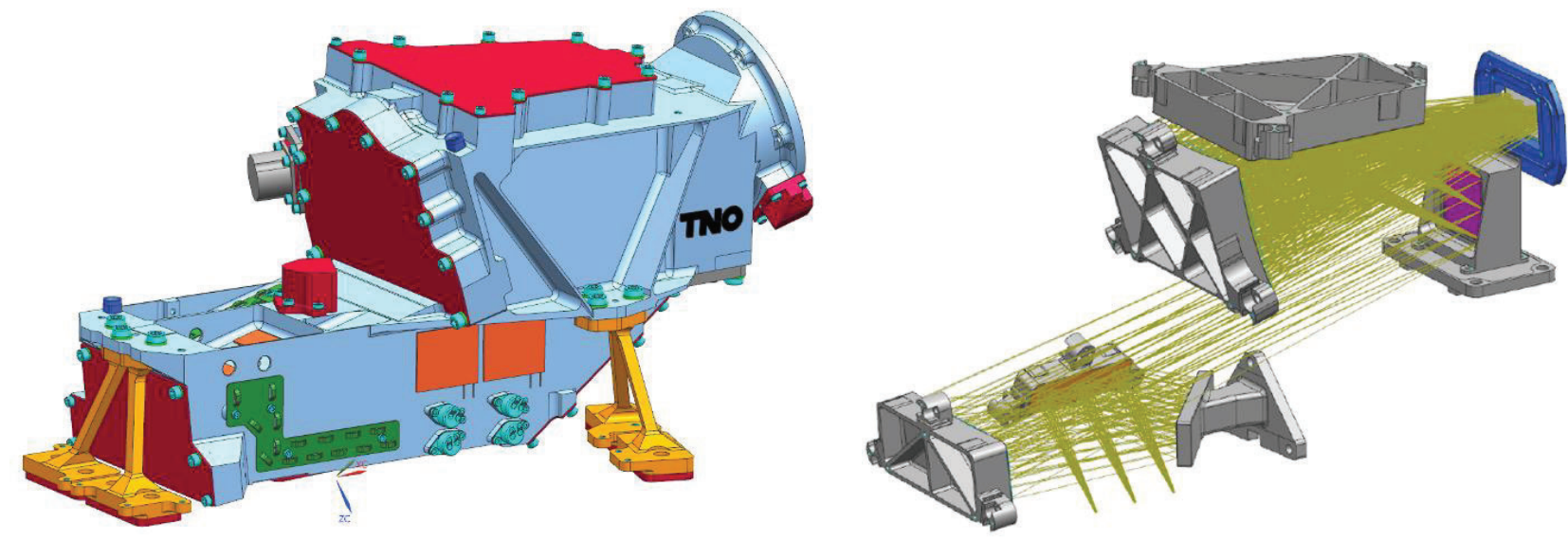

Figure 12. Overview of the mechanical design of the UV1-spectrometer. The optical components and flexural mounts are bolted to the housing. The housing is kinematically connected to the satellite frame. 


\section{Mechanical integrity}

The UV1 spectrometer is designed to survive the loads and environmental conditions during launch and in orbit. A maximum quasi static load of $25 \mathrm{~g}$ is applicable for the UV1. A random vibration spectrum of maximum $9.7 \mathrm{~g}$-RMS applies, as well as shock loads up to $500 \mathrm{~g}$ : see Table 3 .

To proof the numerical analysis, shock tests have been performed on a dedicated UV1 shock model that contains exact copies of the most critical flight hardware components; the UV1-housing, the struts, the grating, the CAM2 mirror and the FSCP (including its mount). For the other components, representative dummy masses were used. The shock tests have been performed at Astro- und Feinwerktechnik, Berlin, Germany using an impact hammer setup (see Figure 13, left). Note that a $1.5 \mathrm{~kg}$ mass dummy is added to the UV1 to represent the actual flight detector. Pre- and post-test Coordinate Measurement Machine (CMM) and theodolite measurements confirmed that the UV1 mechanical design is capable to survive a significant shock loads.

Quasi Static and Random vibration tests have been performed on the UV1 PFM at Centre Spatial de Liège (CSL) in a dedicated ISO class 5 tent to ensure proper cleanliness levels. An $88 \mathrm{kN} \mathrm{LING} \mathrm{shaker} \mathrm{was} \mathrm{used} \mathrm{(see} \mathrm{Figure} \mathrm{13,} \mathrm{right).}$ The mechanical components of the UV1 are bolted together with high preload forces to prevent slippage of the interfaces. As a result the structure shows limited mechanical damping performance; at the main resonances a $\mathrm{Q}$ factor of $>100$ was found. To prevent exceedance of the limit interface loads a few steep notches had to be applied in the applied spectra. The vibration tests revealed that the mode shapes and Eigenfrequencies of the UV1 as predicted by the mechanical model were accurate within a few percent and no further adaptation of the model was needed.

Resonance searches were executed prior and post the random vibration tests. The mechanical resonance frequencies did not shift in the course of the vibration experiments. Comparing pre- and post CMM and theodolite measurements of the structure confirmed that the alignment of the UV1 instrument was preserved.

Finally, the UV1 PFM was subjected to a series of qualification thermal cycles, which showed that the design is able to withstand its limit (non-operational) temperatures without any structural changes or changes in performance.

\section{Mechanical Stability during orbit}

The UV1 structure is exposed to changing environmental conditions during operations. To ensure the performance requirements of the system, thermal fluctuations of the housing should be kept to a minimum. Therefore, heaters at 3 well-chosen locations are attached to the UV1-housing. The setpoint temperature of the UV1 is $20{ }^{\circ} \mathrm{C}$ during all operational conditions. A Structural Thermal Optical Performance analysis was done to show that the UV1 spectrometer meets the stability requirements during orbit. The thermal model accuracy was confirmed with a series of thermal balance tests on the UV1 PFM. 
Table 3. UV1 random vibration loads and shock loads

\begin{tabular}{|l|l|}
\hline \multicolumn{2}{|l|}{ Random Vibration load } \\
\hline Frequency $[\mathrm{Hz}]$ & $P S D[\mathrm{~g} 2 / \mathrm{Hz}]$ \\
\hline 20 & 0.01 \\
\hline 100 & 0.15 \\
\hline 300 & 0.15 \\
\hline 2000 & 0.01 \\
\hline g-RMS & 9.7 \\
\hline
\end{tabular}

\begin{tabular}{|l|l|}
\hline \multicolumn{2}{|c|}{ Shock load } \\
\hline $\begin{array}{l}\text { Frequency } \\
{[\mathrm{Hz}]}\end{array}$ & $\begin{array}{l}\text { Load } \\
{[\mathrm{g}]}\end{array}$ \\
\hline 100 & 30 \\
\hline 270 & 16 \\
\hline 2000 & 500 \\
\hline 10000 & 500 \\
\hline & \\
\hline
\end{tabular}
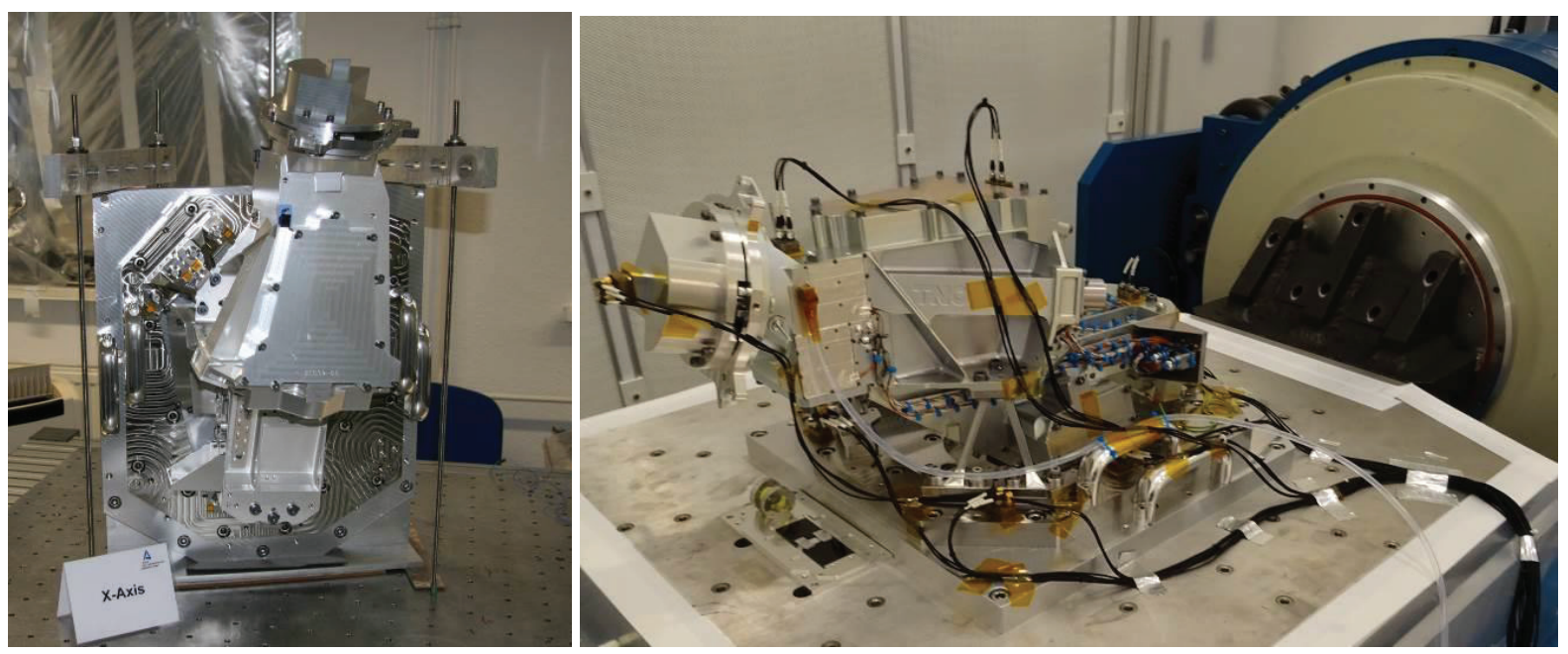

Figure 13. Left: UV1 shock experiment with the impact hammer setup. Right: UV1 PFM on the 88kN LING shaker at CSL

\section{THE UV1 SPECTROMETER PROTO FLIGHT MODEL (PFM) ASSEMBLY, INTEGRATION AND TESTING}

\subsection{Assembly and integration}

The opto-mechanical design of the UV1 system and its components is aimed to be assembled on manufacturing tolerances. Only the FOLD-3 position and the detector plane z-position are optimized to compensate for all the combined tolerances in the components and sub-assemblies. These compensators were chosen to be both effective and accessible to the operator during the alignment procedure. The metric for the alignment is the imaging performance of the UV1 spectrometer; a weighed merit-function of spectral and spatial spot size.

The actual alignment of the PFM showed that the FOLD-3 compensator was not needed and the optimum detector Zposition was found to be just $60 \mu \mathrm{m}$ behind the designed focal plane position.

\subsection{Performance verification approach and equipment}

The performance verification of a standalone spectrometer without telescope, entrance slit, limiting aperture stop and flight detector poses a major challenge. For this verification TNO needed to develop dedicated Ground Support Equipment (GSE) that can be used under both ambient and thermal vacuum conditions.

First, a dedicated optical stimulus reproduces the illumination from the telescope in terms of pointing and f-number. Second, a dedicated GSE Slit Assembly mimics the homogenizer of the telescope subsystem and specifies the spectrometer object planes in a purely mechanical way. Third, a dedicated GSE Test Detector is placed at the image location to determine the image plane orientation and location and to verify the optical performance parameters with a 
high accuracy. Finally, a special Thermal Setup has been designed and build to perform the thermal vacuum tests and to verify the system performance at operational conditions. During the alignment and performance verification the UV1 subsystem is mounted on a dedicated Multi-Purpose Adapter (MPA) plate, that also houses the GSE Slit assembly.

\section{GSE optical stimulus}

The GSE optical stimulus is a hybrid system that employs an (ambient) imager and a stable relay telescope inside vacuum.

The used light source of the stimulus is a tunable OPO laser (EKSPLA NT-242). It uniformly illuminates a rectangular aperture of $68.0 \times 6.0 \mathrm{~mm}$ via a Spectralon coated integrating sphere. The ambient part of the stimulus re-images this rectangular aperture onto the GSE slit with unit magnification. This allows control over the illumination F\# as well as being able to use a reasonably sized polarizer for the polarization characterization of UV1. The F/\# of the system is 9.0 (direction across the slit) by 8.0 (direction along the slit). In combination with an exchangeable aperture this allows both over- ad well as underfilling the entrance pupil of UV1. All performance verification in ambient is done with this stimulus.

The vacuum relay telescope re-images the output of the stimuus on the UV1 GSE slit when it is inside the thermal vacuum chamber. As an additional challenge, it should allow accurate pointing measurement through the system itself using a collimated beam, so that changes in pointing performance due to e.g. temperature changes within the vacuum chamber can be observed from the outside. The target for this pointing measurement is an alignment cube that is positioned next to the GSE slit. This means that the vacuum relay system should have a very small change in angular pointing over the entrance aperture.
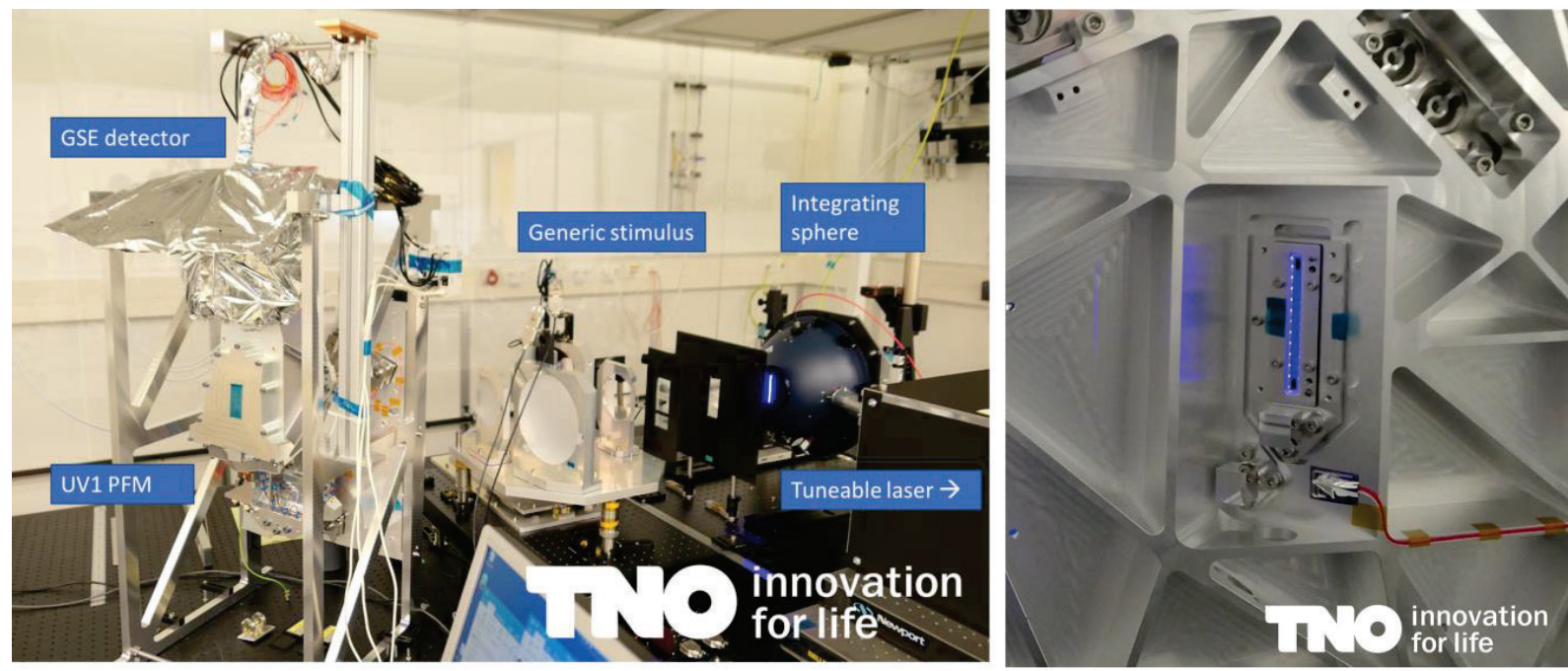

Figure 14. Left: UV1 PFM system set up for alignment and performance verification in ambient. Right: the GSE Slit Assembly illuminated by the GSE Ambient Optical Stimulus (at visible wavelength)

\section{GSE Multi-Purpose Adapter Plate and Slit assembly}

During the alignment, test and performance verification phase the UV1 spectrometer is mounted on a Multi-Purpose Adapter plate (MPA). The purpose of this plate is to provide a stable flight-representative mounting surface for the spectrometer as well as holding the GSE slit assembly in the right place. The MPA is constructed of a single Aluminium plate that has been pocketed to reduce weight. The common flatness of the mounting surfaces is $<25 \mu \mathrm{m}$ so that the induced deformation of the UV1 subsystem is minimal.

The GSE slit assembly mimics this astigmatic object that is presented by the telescope. The "spectral slit" is composed of two sharp wedges that are separated by $0.48 \mathrm{~mm}$. In front of the spectral slit various "spatial slit masks" can be placed. For the main performance verification, a slit mask with a number of sharp, vertical open lines is used. The roughness of the edges of these spatial slits is less than a few micrometers in order not to affect the spatial spot size measurements. 
Both slits are built into a single GSE slit assembly, that also contains an alignment cube to align the optical stimulus exactly along the normal of the slit assembly. The location and orientation of the GSE slit is determined with respect to the mounting references for UV1 on the MPA using a state of the art Coordinate Measurement Machine (CMM). This allows the position of the slit to be measured with an accuracy of several micrometers.

When the slit assembly is illuminated using a single wavelength (from the laser) the result is that UV1 will project a series of squares on the GSE Test Detector, see Figure 15. By analyzing the sharpness of the edges of these squares, the spatial and spectral spot sizes can be determined. By analyzing the positions of the edges, the dispersion, image size and distortions can be measured. Different spatial slit masks can be placed for different types of verifications.

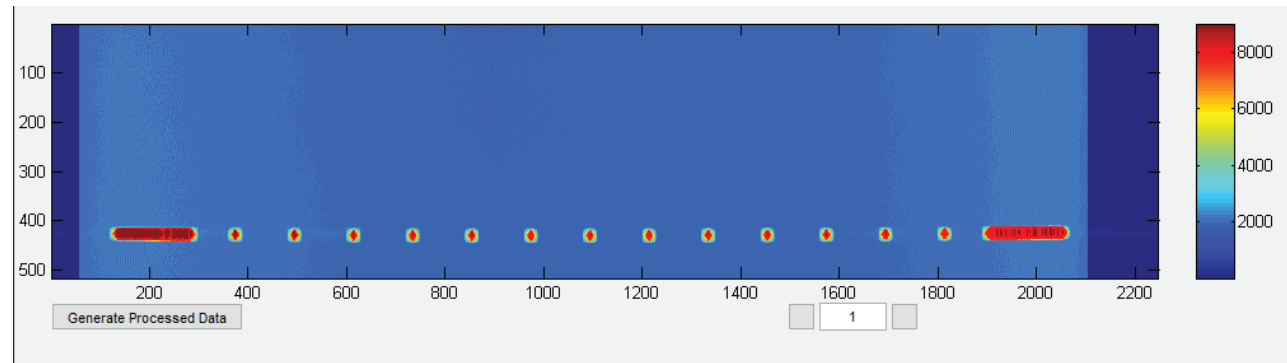

Figure 15: Actual verification measurement of the UV1 PFM that shows the image of a single wavelength as a result of the GSE slit with a spatial mask.

\section{GSE detector}

Since the UV1 spectrometer subsystem does not have a detector, a separate GSE detector had to be built to verify all optical performance requirements in the image plane. As an additional challenge, many of the performance requirements require verification with spatial resolution that is much finer than the flight detector pixels of $20 \times 30 \mu \mathrm{m}$. Combined with requirements for a high quantum efficiency in the UV, a low reflectivity and a design that is vacuum compatible this rules out many potential detector solutions.

A feasible detector candidate was found to be the E2V CCD42-10 Back Illuminated High Performance AIMO CCD Sensor. This CCD detector and the accompanying (vacuum compatible) front-end electronics for read-out and temperature control was delivered by the University of Leicester, United Kingdom. The CCD meets all requirements, except that its size in spectral direction $(6.9 \mathrm{~mm})$ does not cover the entire UV1 spectral image size. Therefore, the GSE detector assembly in which it is mounted has the option to shift the CCD in spectral direction. Furthermore, it has three actuators, which allows for automatic and precise scanning of the detector in $\mathrm{z}$-direction and rotation around the $\mathrm{x}$ and $\mathrm{y}$ axes. Figure 16 shows a photo of the GSE Detector Assembly. 

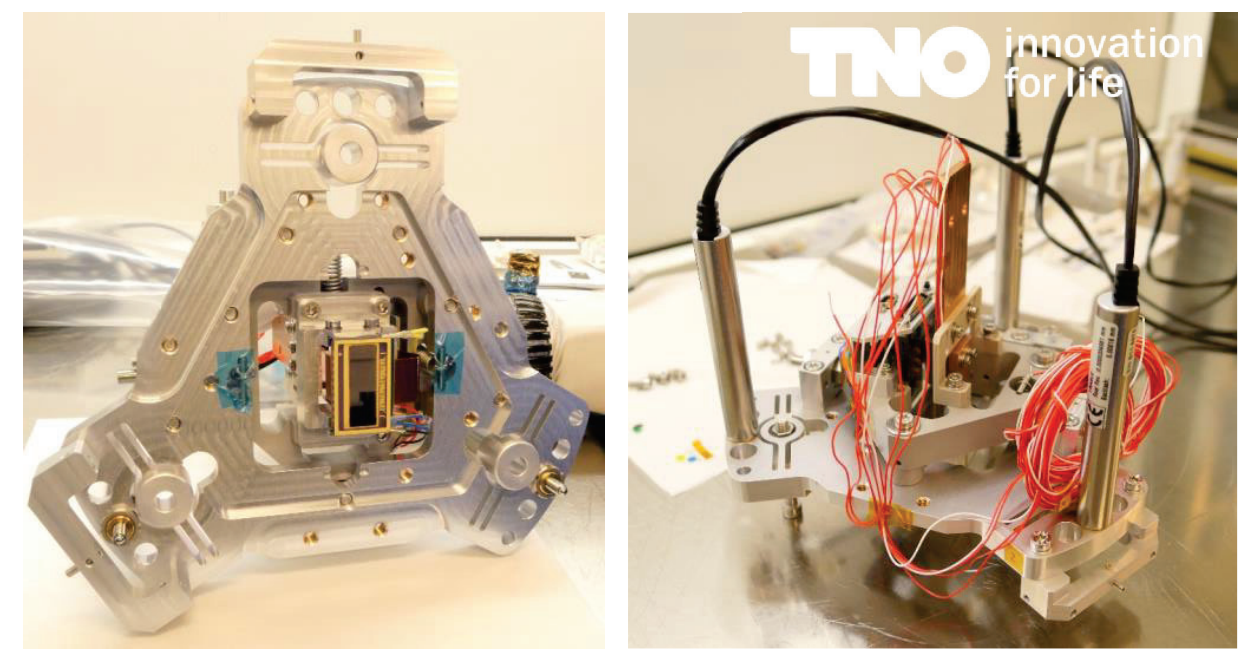

Figure 16. The UV1 GSE Test Detector assembly

\section{Thermal test setup}

For the thermal vacuum testing of the UV1 subsystem as well as the operational performance verification, a dedicated thermal setup has been built in TNO's Vacuum Calibration Facility (VCF). The VCF is a vacuum vessel of $1.5 \mathrm{~m}$ in diameter dedicated for testing and calibrating optical space instruments. However, the thermal stability of the thermal shroud of this vessel is not enough to meet the stringent verification accuracy that is required. Therefore, the UV1 instrument was placed inside an enclosure of 6 panels that each are heated to the prescribed radiative interface temperature of $5^{\circ} \mathrm{C}$ that the system will see. A photo of the thermal vacuum test setup inside the TNO VCF can be seen in Figure 17.

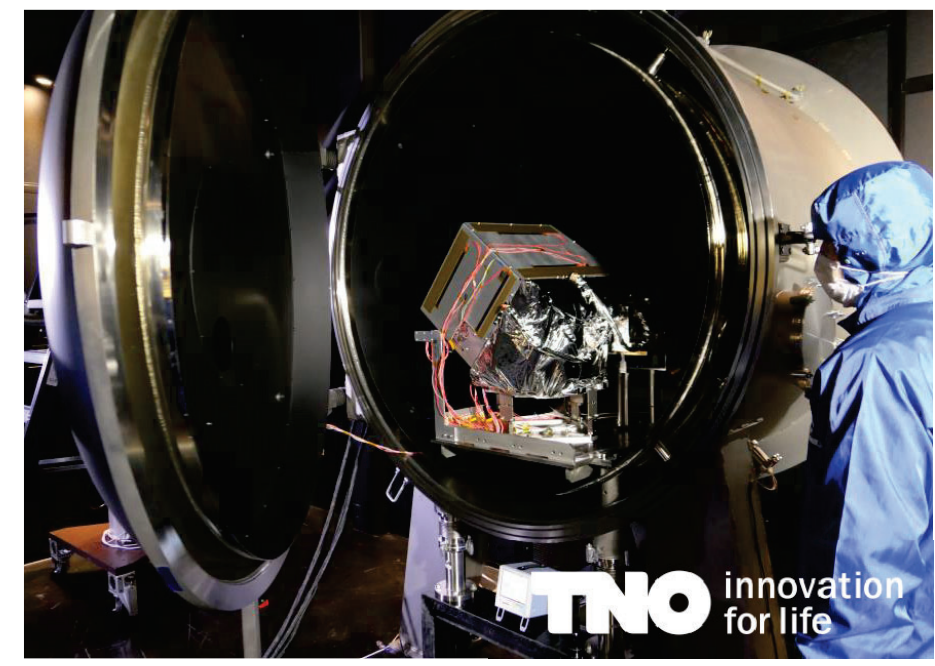

Figure 17. The UV1 thermal test setup inside the TNO VCF vessel

\subsection{Performance verification results}

The (qualification and) performance verification test campaign of the UV1 PFM was executed between September 2019 and May 2020. All verification tests were performed at TNO in Delft, except for the vibration test which was performed at CSL, Liege, Belgium. 
The stringent optical performance requirements of the UV1 were all met before and after the vibration and thermal cycle tests as well as during the ambient-to-operational environment change. The system performed as expected and well within the tolerated budgets.

The tight requirements on the knowledge (and change) of the reference systems were very hard to verify. In many cases a large set of individual measurements taken with the CMM, theodolites, microscopes and on the CCD had to be combined, including all their coordinate transformations and measurement uncertainties. A dedicated Matlab repository of scripts and functions was created for this task. The majority of the position uncertainty requirements could be met, but for the rotations this was much harder, mainly because of the limited size of some mechanical components.

The measurement of the stray light proved to be the most challenging of all verification measurements, mainly because of the $>3$ orders of magnitude range in irradiance levels. To directly verify the requirements by test would mean that individual stray light measurements with an $\mathrm{SNR}>1 \mathrm{E}+6$ were required, which was unfeasible within realistic measurement times using the selected detector. Instead an indirect stray light verification method was chosen based on a combination of simulation and as-built component level data. The manufactured mirrors have a microroughness between $0.4 \mathrm{~nm}$ and $1 \mathrm{~nm}$ rms, with 4 out of 5 mirrors having a lower roughness than the anticipated $0.7 \mathrm{~nm}$. Despite the complex surface form, the as-built gratings delivered by Zeiss exhibit excellent scattering properties equivalent to $1.8 \mathrm{~nm} \mathrm{rms}$ roughness. The resulting in-band and out-of-band stray light fractions of the UV1 PFM were $\leq 1.7 \%$ and $\leq 1.2 \%$ respectively, which is considered a very good performance for a design with aluminium mirrors and a holographic grating over this wavelength range.

The actual stray light verification tests were able to achieve an SNR of about 3000 and were used to quantitively verify the simulation results at a number of wavelengths with success.

\subsection{Conclusion and outlook}

The performance verification campaign of the UV1 was almost performed on schedule, even though the circumstances in the second half of the campaign were less than optimal due to the COVID-19 restrictions. To a large extent this is attributed to the extensive preparation efforts of the campaign and the GSE.

In July 2020, the UV1 PFM successfully passed the qualification and acceptance review and it was delivered to Airbus as the first optical subsystem of the Sentinel-5 instrument.

Currently the performance verification of the second UV1 flight model (FM2) is ongoing, together with the final AIT activities of the third flight model (FM3). The delivery of these models is scheduled in 2021 and 2022 respectively

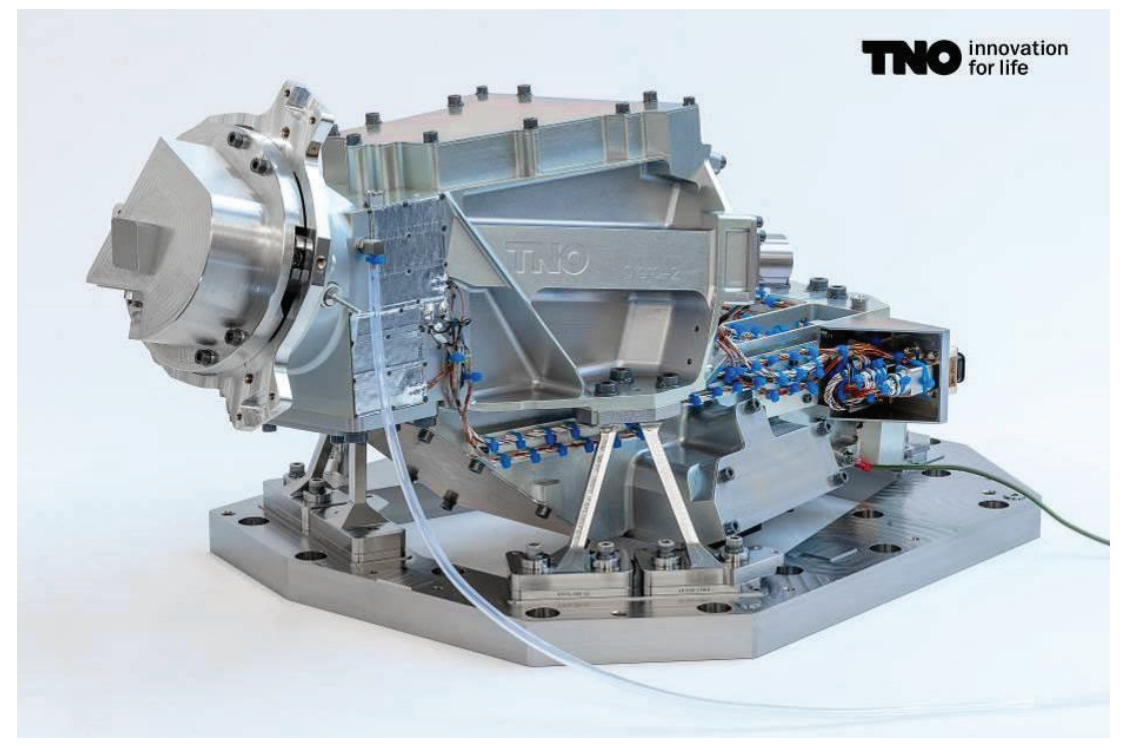

Figure 18: photo of the UV1 Proto Flight Model (with a mechanical dummy at its FPA interface) on its Mechanical Adapter. 


\section{ACKNOWLEDGEMENTS}

The authors would like to thank the whole Sentinel-5 project team, as well as all our industrial partners for their fruitful cooperation in this challenging project. The authors would like to give special thanks to our industrial partners at TNO in Delft, Sodern in Paris, Jena Optronics, Leonardo in Florence for the inputs and contribution of figures to this article.

The Sentinel-5 project is funded by European Commission under an ESA contract, as part of the Space Component of the Copernicus Program.

\section{REFERENCES}

[1] Perez Albinana, Abelardo et al., "Sentinel-5: the new generation European operational atmospheric chemistry mission in polar orbit", Proc. SPIE 10403, Infrared Remote Sensing and Instrumentation XXV (2017).

[2] Irizar, J., Melf, M., Bartsch, P., Koehler, J. " Sentinel-5/UVNS", Proc. SPIE 11180, 1118004-1 (2018).

[3] Meister, C., Bauer, M., Keim, C., and Irizar, J., " Sentinel-5/UVNS instrument: the principle ability of a slit homogenizer to reduce scene contrast for earth observation spectrometer", Proc. SPIE 10423, 104231E (2017).

[4] Brukhardt, M. et al, "Manufacturing method for monolithic freeform Offner-gratings for hyper-spectral imaging", Proc. SPIE 11151, Sensors, Systems, and Next-Generation Satellites XXIII (2019).

[5] Weber, T. et al, "UV coatings by IAD and PARMS technology for Sentinel-5 mission", ICSO 2020 P073 (2020) 